\title{
Arbor
}

\section{Quo Vadis: el español y el Hispanismo en Sudáfrica}

\section{Cathy Maree y Carmen Sánchez Martín}

Arbor CLXVIII, 664 (Abril 2001), 441-460 pp.

Nuestro objetivo en este ensayo es presentar la situación del español y del hispanismo en el complejo mundo que ha sido y sigue siendo Sudáfrica. Con el fin de entender el papel que desempeña el español en el África austral, se traza la historia socio-lingüística sudafricana, la remota y la reciente, como base indispensable para el posterior análisis comparativo del español y las otras lenguas y culturas extranjeras estudiadas en el país. El enfoque entonces pasa a contestar a esta pregunta: ¿Cuál es el presente y futuro del español en Sudáfrica, país multicultural, multirracial, multilingüístico?

\section{Introducción y planteamiento del problema}

La situación del español como lengua extranjera en Sudáfrica a primera vista deja a uno perplejo. Sudáfrica es una sociedad pluricultural y multilingüe, cuyos habitantes, más de cuarenta millones, suelen hablar dos o más de las once lenguas oficiales. Desde el año 1994, fecha en la que se celebraron las primeras elecciones democráticas del país, la política del gobierno del Congreso Nacional Africano (ANC) ha sido promover el multilingüismo en todos los sectores de la vida pública y, fundamentalmente, en el entorno escolar. En el boletín oficial del gobierno sobre el papel de las lenguas en la educación, figura como uno de los objetivos el de apoyar la enseñanza y el aprendizaje de todas las lenguas requeridas en Sudáfrica, Aincluso las que son importantes para el comercio y la comunicación internacional» (Government Gazette, 1997:4, traducción nuestra). Requiere, incluso, que en los dos últimos años de la enseñanza secundaria Aes obligatorio que los alumnos estudien y se 
examinen en dos lenguas, de las que una tiene que ser una lengua oficial del país» (Government Gazette, 1997:4, traducción nuestra). Sin embargo, consta que el español no se beneficia de esta política gubernamental en pro de las lenguas, tanto oficiales como extranjeras. Como disciplina universitaria sólo se imparte en una de las veintiuna universidades del país y en los últimos dos años el número no sobrepasa un total de doscientos estudiantes. A nivel preuniversitario no aparece como asignatura oficial, es decir, no figura como disciplina subvencionada por el Ministerio de Educación aunque sí, hecho no usual en España, un alumno puede presentarse al examen oficial preuniversitario en español (el equivalente de la «selectividad» en España). A lo largo de los años, el número de estos candidatos no ha superado los quince. Las academias y escuelas de lengua privadas, tampoco registran un número extraordinario de alumnos de español.

¿Qué significa todo lo anterior? ¿Existe un marcado desinterés en cuanto al mundo hispánico por parte de los sudafricanos? ¿No hay interés en el idioma español? ¿Es desprestigiada con relación a otras lenguas de raigambre internacional, con relación al inglés por ejemplo, o al francés $\mathrm{y}$ al portugués, idiomas éstos hablados en África? ¿O podríamos incluir otros factores? ¿Qué se puede hacer para transformar esta situación? ¿Qué es lo que se propone para mejorarla?

Con el fin de contestar estas preguntas, creemos conveniente hacer un repaso histórico de la situación y las políticas lingüísticas en Sudáfrica que pueden haber influido en el estudio de idiomas extranjeros en el pasado, para contrastarlas con la política y tendencias de hoy. De ahí nos centraremos en la historia de la enseñanza del español en el país como base para nuestras perspectivas sobre futuros proyectos cuya finalidad es promover el hispanismo en este sur profundo del continente africano.

\section{La situación lingüística en Sudáfrica: desde la época precolonial hasta el presente}

En la época precolonial tardía —antes de 1652, año que vio el asentamiento de los primeros colonos europeos en lo que es hoy Sudáfricahabía diversos pueblos africanos agrupados en reinos y hasta comunidades nómadas, todas sin políticas ni prácticas de dominación establecida. Existía una alta inteligibilidad lingüística entre las distintas familias de lenguas indígenas habladas, lo cual llevó a un alto grado de osmosis. 


\section{Quo Vadis: el español y el Hispanismo en Sudáfrica}

La época del colonialismo temprano - 1652 a 1909- vio la introducción e imposición primero del holandés (1652-1795) y después del inglés (1795-1909), lenguas que desplazaron de su espacio social las hablas indígenas africanas. El proceso político asumió características lingüísticas, es decir, las luchas por el poder entre los angloparlantes y los de habla afrikaans, idioma que evolucionó en Sudáfrica del holandés del siglo XVII, produjeron divisiones y antagonismos lingüísticos.

Durante la época colonial tardía -1909 a 1947- el dominio del inglés repercutió negativamente en las otras lenguas habladas en el país. La creciente urbanización del africano negro resultó en su adopción del idioma inglés por ser considerado la lengua asociada al ascenso socio-económico; el inglés se convirtió en la lengua de prestigio. Por consiguiente, las lenguas indígenas sufrieron una marcada marginación contrastando con el avance de la lengua afrikaans que comenzó a beneficiarse del fervor del movimiento nacionalista de los afrikaners, quienes lucharon por el reconocimiento del afrikaans como lengua oficial.

La época del «apartheid» -la segregación oficial de razas desde 1948 a 1994- presenció el dominio del afrikaans en todos los sectores de la vida pública y su creciente insinuación en la vida económica, aunque nunca a expensas del inglés que mantenía su posición dominante en el mundo del comercio internacional. El auge del nacionalismo afrikaans de este período llevó a la creación de nuevas divisiones lingüísticas en el país que también impactaron en la enseñanza: el intento del gobierno de imponer el afrikaans como medio de instrucción en los colegios secundarios negros originó la trágica revuelta de Soweto en 1976, que fue uno de los hitos en el camino hacia el ocaso del apartheid.

En la época post-apartheid —desde 1994- existe la igualdad estatuaria entre las once lenguas oficiales: inglés, afrikaans y las nueve lenguas indígenas bantúes. También se ha legislado la promoción de las lenguas indígenas, junto con la de las lenguas de legado cultural las lenguas europeas e índicas habladas por las comunidades históricamente inmigrantes en Sudáfrica-. El dominio del inglés -lengua materna de sólo el $9 \%$ de la población-sigue en todos los sectores públicos y en los medios de comunicación. Esto de nuevo va repercutiendo negativamente en el afrikaans - hablado hoy por el 15\% de la población-y hasta cierto punto en las lenguas indígenas mayoritarias cuyos líderes intelectuales y políticos se expresan públicamente casi siempre en inglés.

Nuestro resumen ${ }^{1}$, aunque por fuerza breve y simplificado, descubre que no se puede separar la situación lingüística del país durante los últimos cuatrocientos años de las políticas de colonización y segregación 
racial. Durante los cuarenta y seis años del régimen de apartheid entre 1948 y 1994 no se promovió el aprendizaje de idiomas extranjeros. En efecto, fue todo lo contrario: para contrarrestar el auge natural del inglés, los líderes del gobierno aumentaron el prestigio del afrikaans, una lengua confinada a hablantes dentro de Sudáfrica. La política de entonces fue crear una sociedad bilingüe, en la que el concepto del bilingüismo se limitaba a las lenguas afrikaans e inglés. Sin embargo esta misma política no permitió a la población negra el acceso al inglés. Aunque el motivo declarado de impartir la educación primaria en las lenguas indígenas fue el de conservarlas, el sentimiento para la gran mayoría de los negros sudafricanos fue el de limitarles la comunicación con otras culturas y pueblos, tanto dentro como fuera del país. Como consecuencia de esta política, la mayoría de la población negra sudafricana actual tiene como lengua primera y segunda y hasta tercera un idioma indígena (Webb, 1995:18). Este estado de multilingüismo es algo que el gobierno del ANC no quiere sacrificar. Prefieren distanciarse de la política de asimilación lingüística de los países francófonos y lusófonos de África, cuyos ciudadanos alfabetos tienen como primera lengua el francés o el portugués.

En la actualidad existe una tensión marcada entre la política del multilingüismo y su práctica en los colegios a lo largo de Sudáfrica. En una encuesta realizada por David Meyer en 1998, se observa que en los colegios situados en las áreas con una población mayoritariamente negra, tanto los alumnos como sus padres y sus profesores eligen el inglés como medio de instrucción, en lugar de su lengua materna. Sin embargo, el sondeo también revela que en la práctica el 67,5\% de los profesores y el $77,5 \%$ de los alumnos dicen que emplean su lengua materna en las aulas, sobre todo para la comunicación verbal (Meyer, 1998:16).

De acuerdo con este contexto, se puede prever que la enseñanza y el aprendizaje de lenguas extranjeras en Sudáfrica no superarán en un futuro próximo la preferencia por el inglés como segunda u otra lengua entre la población mayoritaria. Y por encima, otra encuesta parece confirmar que para demasiados sudafricanos negros «aprender una lengua extranjera es más que nada hacer traducciones del inglés a esa lengua» (de Kadt 1999:18, traducción nuestra). En otras palabras aún persiste la noción de que el acceso a una lengua extranjera es únicamente por medio del inglés.

¿Cuáles han sido y siguen siendo las implicaciones de la compleja situación lingüística sudafricana para las lenguas extranjeras? Primero daremos una relación del papel general de estas lenguas para luego pasar a examinar la situación del español en Sudáfrica. 


\section{La situación histórica y actual de lenguas extranjeras en Sudáfrica}

En este apartado ojeamos más bien en forma diagramática y estadística la enseñanza de idiomas extranjeros en Sudáfrica para poder contextualizar mejor la situación del español en el apartado siguiente. Uno de los factores que influye en el interés en aprender una lengua extranjera es la presencia de una comunidad de hablantes nativos en el país en cuestión. En Sudáfrica, aparte de las raíces coloniales de origen inglés y holandés como arriba mencionamos, existen numerosos grupos de hablantes de alemán, portugués, e italiano. El hecho de que exista un examen de alemán como primera lengua o lengua materna dentro de los exámenes oficiales preuniversitarios en Sudáfrica es indicio de la importancia numérica de esta comunidad $\left(400.000^{2}\right)$. El portugués se considera una lengua comunitaria sudafricana o lengua heredada, dado que es hablada por unas 600000 personas en este país, la mayoría procedente de los países vecinos Mozambique, Angola, las islas Cabo Verde y Madeira. La comunidad italiana (70.000), aunque ya no tan numerosa en comparación con las dos mencionadas, continúa ejerciendo una considerable influencia económica y cultural en Sudáfrica. En cambio, como comenta Paloma Lapuerta (1997:490), el español en Sudáfrica no goza de raíces coloniales de España u otro país hispanohablante, ni de la presencia hispanoparlante de una fuerte comunidad inmigrante (en la actualidad hay 1.500 españoles y 4.300 hispanoamericanos residentes en Sudáfrica). Estos factores han repercutido favorable o negativamente en el interés por las lenguas extranjeras en Sudáfrica, sobre todo a nivel preuniversitario.

Otros factores que han motivado la introducción de lenguas extranjeras en el sistema educativo sudafricano son las afiliaciones religiosas y culturales. El árabe moderno estándar ${ }^{3}$ es materia estudiada principalmente por la comunidad musulmana (aproximadamente 800.000) aunque también por personas que comercian con los países árabes y por diplomáticos sudafricanos; el hebreo moderno, por la comunidad judía (100.000) asentada en Sudáfrica desde principios de este siglo; el holandés, principalmente por la comunidad afrikaner como parte del currículo universitario del afrikaans; el griego, por la comunidad griega inmigrante en Sudáfrica (80 000); y las cinco lenguas índicas (gujarati, hindi, tamil, telugu y urdu), por los pueblos indios (1.000.000), cuyos antepasados llegaron a Sudáfrica en el siglo pasado como mano de obra barata o como comerciantes.

Por último, la importancia de una lengua por motivos de su prestigio internacional o por los lazos comerciales y políticos con Sudáfrica también ha influido y continúa influyendo en su aceptación como materia digna de es- 
tudio. El francés goza de una larga tradición como lengua internacional y más aún como lengua hablada en varios países de África; el español va ganando más y más prestigio como lengua internacional y, como veremos más adelante (5.1), aumentan los lazos comerciales y políticos con España e Hispanoamérica; y se considera importante el chino no sólo por la histórica comunidad china inmigrante en Sudáfrica (50.000) sino también por el gran mercado que representa en el mundo comercial. Sin embargo, a pesar de que Japón es el segundo país importador/exportador de Sudáfrica, el japonés aún no es estudiado en los colegios o universidades sudafricanas.

\subsection{En las universidades}

A nivel universitario el cuadro siguiente revela cuándo fue establecida cada lengua como disciplina (materia que se imparte hasta el nivel postgrado), en cuántas de las veintiuna universidades sudafricanas se enseña actualmente, en qué universidades ha sido eliminada y el número de alumnos matriculados en 1970, 1990 y 1998 (número que incluye a los alumnos de pre y posgrado, aunque éstos son relativamente escasos). Conviene aclarar que no aparece el holandés en nuestra lista aunque se enseña en muchas universidades sudafricanas. Como ya hemos mencionado, forma parte del currículo de los departamentos de afrikaans y por lo tanto no se imparte como lengua extranjera.

La eliminación de muchas de estas lenguas como disciplinas en algunas de las universidades más prestigiosas del país (cuarta columna) y la reducción severa del número de cursos ofrecidos responden a una serie de reestructuraciones impuestas en parte por los cortes de presupuesto del gobierno - todas las universidades acreditadas en Sudáfrica son públicas- y en parte por el bajo número de estudiantes matriculados en estas materias. En la tabla (columnas cinco a siete) se observa un descenso progresivo en el número de estudiantes matriculados en casi todas las lenguas extranjeras. Esto es una causa de preocupación y, como postula Paloma Lapuerta, su explicación hay que buscarla en varios hechos: «el cambio de una población universitaria de origen principalmente europeo a una población universitaria creciente de origen africano, que no tiene motivos emocionales ni, por ahora, profesionales para estudiar las lenguas europeas; la prioridad del estudio del inglés para los no angloparlantes...; y la deficiente preparación intelectual ... de la gran mayoría de los estudiantes que están accediendo recientemente a las universidades sudafricanas ...» (1997:489). Es verdad que tardará varios años hasta que las consecuencias del antiguo sistema educativo del apartheid y de los años de aislamiento de Sudáfrica vayan desapareciendo. 
Quo Vadis: el español y el Hispanismo en Sudáfrica

\begin{tabular}{|c|c|c|c|c|c|c|}
\hline \multirow[t]{2}{*}{ Lengua } & \multirow{2}{*}{$\begin{array}{l}\text { Comienzo/ } \\
\text { Universidad }^{4}\end{array}$} & \multirow{2}{*}{$\begin{array}{l}\text { Universidades } \\
\text { actuales }^{4}\end{array}$} & \multirow{2}{*}{$\begin{array}{l}\text { Cierre/ } \\
\text { Universidad }^{4}\end{array}$} & \multicolumn{3}{|c|}{ Número de estudiantes } \\
\hline & & & & 1970 & 1990 & 1998 \\
\hline Alemán & $\begin{array}{l}1829 \text { (En el } \\
\text { colegio que fue } \\
\text { precursor de } \\
\text { la UCT) } \\
\text { UP,UPE,US, } \\
\text { Unitra, WITS) }\end{array}$ & $\begin{array}{l}13 \text { (PUCHE, } \\
\text { RAU, RU,UCT, } \\
\text { UND,UNP, } \\
\text { Unisa, UOFS, }\end{array}$ & $\begin{array}{l}\text { UZ (1999) } \\
\text { UDW (1998) }\end{array}$ & 1787 & 1376 & 920 \\
\hline $\begin{array}{l}\text { Árabe } \\
\text { moderno } \\
\text { estándar }\end{array}$ & 1963 (Unisa) & $\begin{array}{l}3 \text { (Unisa, } \\
\text { UWC, RAU) }\end{array}$ & UDW (1998) & 88 & 216 & 133 \\
\hline $\begin{array}{l}\text { Chino } \\
\text { Mandarín }\end{array}$ & 1993 (Unisa) & 2 (Unisa, US) & & - & - & 64 \\
\hline Español & 1966 (Unisa) & 1 (Unisa) & UND (1998) & 45 & 126 & 145 \\
\hline Francés & $\begin{array}{l}1829 \text { (En el } \\
\text { colegio que fue } \\
\text { precursor de } \\
\text { la UCT) } \\
\text { UOFS, UP, } \\
\text { UPE,US, WITS) }\end{array}$ & $\begin{array}{l}14 \text { (PUCHO, } \\
\text { RAU, RU, UCT } \\
\text { UN, UND, UNP } \\
\text { Unisa, Unitra, }\end{array}$ & $\begin{array}{l}\text { UZ (1999) } \\
\text { UDW (1998) }\end{array}$ & 1565 & 1482 & 1563 \\
\hline $\begin{array}{l}\text { Griego } \\
\text { moderno }\end{array}$ & 1982 (RAU) & 1 (RAU) & WITS (1992) & 56 & 87 & 81 \\
\hline $\begin{array}{l}\text { Hebreo } \\
\text { moderno }\end{array}$ & 1861 (UCT) & 2 (UCT, Unisa) & $\begin{array}{l}\text { WITS (1996) } \\
\text { UND (1994) }\end{array}$ & 243 & 229 & 131 \\
\hline Italiano & 1921 (WITS) & $\begin{array}{l}3 \text { (UCT, Unisa, } \\
\text { WITS) }\end{array}$ & $\begin{array}{l}\text { US (‘92) } \\
\text { RU('90) } \\
\text { RAU ('90) } \\
\text { UOFS ('89) }\end{array}$ & 384 & 286 & 155 \\
\hline $\begin{array}{l}\text { Lenguas índicas: } \\
\text { gujarati, } \\
\text { hindi, tamil, } \\
\text { telu-gu, urdu }\end{array}$ & 1972 (UDW) & 0 & UDW (1998) & $\begin{array}{c}170 \\
(1972)\end{array}$ & 150 & 20 \\
\hline Portugués & 1932 (WITS) & 1 (Unisa) & $\begin{array}{l}\text { WITS (1995) } \\
\text { UCT (1992) }\end{array}$ & 97 & 135 & 90 \\
\hline Ruso & 1960 (Unisa) & 1 (Unisa) & WITS (1994) & 32 & 114 & 43 \\
\hline
\end{tabular}

A otro nivel, Sudáfrica también cuenta con dieciséis institutos politécnicos, llamados Technikons, los cuales ofrecen diplomaturas en una amplia gama de áreas, principalmente de orden vocacional. En los pro- 
gramas de hostelería y turismo, por ejemplo, se incluyen algunos cursos en lenguas extranjeras. Hasta la fecha los idiomas más estudiados son el alemán y el francés; el español es ofrecido en sólo un Technikon.

\subsection{A nivel secundario}

En el año 1873 se iniciaron los exámenes preuniversitarios con el establecimiento de la Universidad del Cabo de Buena Esperanza como única autoridad examinadora nacional. Aquel año aparecieron las lenguas francesa, alemana y holandesa como asignaturas. En el caso del holandés, hay que tener en cuenta que fue, junto con el inglés, una lengua oficial nacional en Sudáfrica hasta 1925, año en que fue desplazada como lengua oficial por el afrikaans. A partir de la creación de la Junta Nacional de Exámenes Preuniversitarios (JMB) en 1918, un alumno podía examinarse de una lengua extranjera de acuerdo con los criterios de esta Junta respecto a la disponibilidad de examinadores y un número suficiente de candidatos. Los exámenes en español comenzaron con regularidad a partir del año 1966.

En la tabla siguiente figuran el número de los colegios en donde hay enseñanza oficial y extra-oficial de cada lengua (por extra-oficial nos referimos a la lengua como materia que se imparte por profesores particulares contratados por el colegio o por los propios padres del alumno) y el número de alumnos en 1970, 1980, 1990 y 1998 que se han inscrito en los exámenes nacionales preuniversitarios. Se notará que en algunos casos - chino, griego y ruso- la lengua se enseña pero no es examinada oficialmente en Sudáfrica. El guión indica los casos en que la lengua o no se enseñó o no fue materia de examen en el año en cuestión.

Con la excepción del portugués y del árabe, las otras lenguas o han mantenido un equilibrio con respecto al número de sus alumnos -el francés y el hebreo moderno- o demuestran un marcado descenso, el caso del alemán e italiano. El español señala un pequeño aumento (en 1999 hay 16 alumnos) pero los números siguen siendo muy bajos. Aunque no hemos podido acceder a toda la información correspondiente, nos consta que, igual que en el sector universitario, han desaparecido muchas lenguas extranjeras del currículo de los colegios privados y estatales en Sudáfrica durante la década de los noventa. Para citar sólo un ejemplo, el francés y el alemán se enseñan en los colegios públicos en sólo tres de las nueve provincias sudafricanas. Como la enseñanza secundaria no es únicamente la responsabilidad del gobierno central sino que también es competencia de las autoridades provinciales, parece que la política gubernamental nacional de promover el aprendizaje de lenguas 


\section{Quo Vadis: el español y el Hispanismo en Sudáfrica}

extranjeras a la que aludimos al principio de este artículo, no es aplicada a nivel provincial. La nueva regulación que exige un mínimo de 30 alumnos antes de que cualquier asignatura reciba una subvención de la provincia ya ha desfavorecido la enseñanza de lenguas extranjeras en muchos colegios secundarios en Sudáfrica.

\begin{tabular}{|c|c|c|c|c|c|}
\hline \multirow[t]{2}{*}{ Lengua } & \multirow{2}{*}{$\begin{array}{l}\text { Colegios Privados } \\
\text { y Estatales }\end{array}$} & \multicolumn{4}{|c|}{ Número de alumnos } \\
\hline & & $1970^{5}$ & $1980^{5}$ & $1990^{5}$ & $1998^{5}$ \\
\hline Alemán & 158 & 2482 & 3408 & 2135 & 1394 \\
\hline $\begin{array}{l}\text { Árabe modelo } \\
\text { estándar }\end{array}$ & 25 & - & - & 75 & 204 \\
\hline Chino mandarín & 77 & - & - & - & - \\
\hline Español & 9 & 2 & 8 & 5 & 12 \\
\hline Francés & 160 & 1034 & 1596 & 1295 & 1207 \\
\hline Griego moderno & 2 & - & - & - & - \\
\hline Hebreo moderno & 18 & 218 & 334 & 251 & 257 \\
\hline $\begin{array}{l}\text { Lenguas índicas: } \\
\text { hindi } \\
\text { gujarati } \\
\text { tamil } \\
\text { urdu }\end{array}$ & $\begin{array}{c}15 \\
2 \\
4 \\
7\end{array}$ & $\frac{-}{-}$ & $\frac{-}{-}$ & $\begin{array}{l}- \\
-\end{array}$ & $\begin{array}{l}25 \\
17 \\
19 \\
25\end{array}$ \\
\hline Italiano & 15 & 34 & 27 & 46 & 18 \\
\hline Portugués & 88 & 49 & 40 & 75 & 322 \\
\hline Ruso & 11 & - & - & - & - \\
\hline
\end{tabular}

\subsection{Institutos/Academias de lengua}

Quienes quieren o tienen que aprender una lengua extranjera en Sudáfrica fuera de los centros universitarios y escolares tienen acceso a una variada gama de posibilidades, desde la opción semi-oficial hasta la privada.

La Escuela Diplomática de Lenguas del Ministerio de Asuntos Exteriores ofrece programas a lo largo del año a sus cadetes y oficiales destinados a las misiones diplomáticas sudafricanas en el extranjero. Imparte cursos desde el nivel inicial hasta el intermedio en alemán, árabe moderno, francés, español y portugués. De acuerdo con sus estadísticas más recientes, la lengua que más se estudia es el francés, debido al alto 
número de países francófonos en África con representación diplomática sudafricana.

La Escuela Militar de Lenguas del Ministerio de Defensa imparte cursos en las siguientes lenguas, desde el nivel inicial hasta el nivel intermedio: alemán, español y francés. Los estudiantes son aquellos oficiales que van destinados a las misiones diplomáticas sudafricanas como agregados militares. Para ellos la lengua más estudiada es el francés.

Como parte de sus programas de extensión universitaria o educación continua para adultos, son tres las universidades sudafricanas que ofrecen cursos de lengua: Unisa, WITS y UCT. Todos son cursos de corta duración (de un mes a cuatro meses) y se centran en el nivel inicial. Las lenguas que ofrecen son: alemán, francés, español, italiano, portugués, ruso $\mathrm{y}$, en WITS, el idioma de signos para sordomudos. El número de estudiantes en cada lengua se limita a unos diez o puede llegar hasta quince por sesión y/o curso. Según los directores de estos programas, el idioma más estudiado es el francés.

La enseñanza de alemán, francés, italiano y portugués cuenta con escuelas subvencionadas por los gobiernos alemán (Goethe Institut), francés (Alliance Française), italiano (Dante Alighieri) y portugués (Instituto Camões). Estos centros se encuentran en las principales ciudades del país. Tres de ellos preparan a sus alumnos para los exámenes oficiales de competencia comunicativa en sus respectivas lenguas: en alemán el Zertifikat Deutsch als Fremdsprache (ZDaF); en francés, el Diplome en la Langue Française (DELF); en portugués, el diploma Português Língua Extranjera (PLE). La Alliance Française ha establecido escuelas en las grandes urbanizaciones negras de Soweto cerca de Johanesburgo y en Mitchell's Plain cerca de la Ciudad del Cabo, donde el número de alumnos va creciendo. Desafortunadamente, el Instituto de Cervantes no parece tener intención alguna de establecer una casa hispánica en Sudáfrica, aunque sí ofrece los exámenes del Diploma de Español como Lengua Extranjera (DELE, ver 4.4. más adelante).

Por último existen las academias privadas, principalmente en las grandes ciudades del país. Puesto que no existen las conocidas escuelas internacionales como, por ejemplo, la escuela Berlitz, los centros de lengua sudafricanos se han establecido por iniciativa de sus dueños particulares. Según dicen los directores, el número de interesados en la década de los noventa ha registrado un aumento considerable en el inglés como lengua extranjera o segunda lengua y en el zulú, considerado extraoficialmente la lingua franca indígena de los sudafricanos. También señalan que el portugués, alemán y francés ocupan los siguientes pues- 


\section{Quo Vadis: el español y el Hispanismo en Sudáfrica}

tos, y en este orden, principalmente por razones comerciales. Respecto al español, todos aseguran que el número de estudiantes aumenta progresivamente, pero va por épocas. El interés por la lengua se encuentra en los negocios, se aprende la lengua por cuestiones de trabajo, indistintamente para realizarlo con España o Hispanoamérica.

\section{La enseñanza del español en Sudáfrica}

Los datos del apartado anterior señalan que la situación respecto a la enseñanza del español en Sudáfrica no es un hecho para celebrar. Sin embargo, en comparación con la situación de otras lenguas no creemos que sea totalmente negativa. En efecto, en términos relativos, el español ha perdido menos estudiantes en los últimos diez años que casi todas las demás lenguas extranjeras. Tenemos mucha fe en el futuro del hispanismo en este país, pero de esto hablaremos más adelante. Por ahora nos centraremos en la situación histórica y actual.

\subsection{A nivel universitario}

En la actualidad el español como disciplina universitaria sólo se ofrece en la Universidad de Sudáfrica (Unisa). Situada en Pretoria, pero con centros repartidos en todo el territorio nacional, es una universidad de educación a distancia, parecida a la Universidad Nacional de Educación a Distancia (UNED) en Madrid. Unisa es la mayor universidad del país, con más de 130.000 estudiantes distribuidos por todo el sur de África e incluso por el mundo entero. El español se incorporó al Departamento de Lenguas Románicas en 1966 como materia del grado BA (Bachelor of Arts), programa de tres años de estudio equivalente a la Diplomatura Universitaria en el sistema educativo español. Su introducción se debió a la iniciativa del entonces director del Departamento, para quien era indiscutible integrar el español dentro de las disciplinas románicas, y a la creciente prosperidad de la universidad basada en el gran aumento de estudiantes. A partir de 1977 se agregaron los grados de BA Honours, programa de especialización en el español, MA (Master of Arts), titulación que corresponde a los cursos de doctorado y la Memoria de la Licenciatura en España, y D. Litt. et Phil., título de doctor. Los cursos comprenden Lengua Española (principiantes hasta el nivel avanzado), Historia de la Lengua, Traducción y Traductología (con el Departamento de Lingüística), Literatura Española (Si- 
glo de Oro y Siglo XX) e Hispanoamericana (de 1492 hasta el presente) y Civilización de España e Hispanoamérica. El cuerpo docente en la actualidad está formado por tres profesores y un lector-becario procedente de la Agencia Española de Cooperación Internacional (Ministerio de Asuntos Exteriores).

En 1984 el Subdepartamento de Español tomó la iniciativa de instituir el Centro de Estudios Latinoamericanos de Unisa (UCLAS), un órgano adjunto que se dedica a la promoción e investigación de los asuntos de la América Latina. El Centro, dirigido por una sola persona, se encarga de publicar una revista semestral -Unisa Latin American Report-y de apoyar y promover proyectos de investigación en áreas de interés mutuo para Sudáfrica y los países del continente americano. Entre los logros del Centro figuran congresos, coloquios y monografías sobre temas tan diversos como el proceso de la redemocratización y la reconciliación con el pasado, la expresión literaria en el Sur del mundo, la cooperación económica entre países vecinos, el desafío de la urbanización acelerada, entre otros.

Desde 1983 hasta 1997 la University of Natal en Durban (UND) ofreció programas de pre y posgrado en español, semejantes a los de Unisa pero a diferencia de ésta, UND es una universidad presencial. La universidad cerró el subdepartamento de español en 1997, obligando a dos de sus tres profesores a buscar puestos académicos fuera del país. Aludimos anteriormente a los motivos de este cierre.

En algunas universidades sudafricanas se encuentran cursos en los que aparecen distintos aspectos del hispanismo a niveles pre- y postgrado, los cuales no dependen del dominio de la lengua española. Por ejemplo, la colonización de América por los españoles con frecuencia forma parte de los cursos sobre el colonialismo en departamentos de Historia. Muchos departamentos de Ciencias Políticas enfocan sus estudios comparativos en aquellos principios y tradiciones políticas que caracterizan a Sudáfrica y los países hispánicos. Y dentro del área de Relaciones Internacionales la universidad WITS tiene un investigador dedicado exclusivamente a las relaciones entre Sudáfrica y los países de Latinoamérica. En muchos departamentos de literatura inglesa o mundial, se estudian obras de autores hispánicos traducidas al inglés.

$\mathrm{El}$ intercambio de personal académico y estudiantes entre Sudáfrica y los países hispánicos no es frecuente, pero podemos destacar el acuerdo actual del Ministerio de Salud sudafricano con el gobierno cubano, por el que cada año unos setenta estudiantes sudafricanos van a Cuba a comenzar la carrera universitaria en medicina y unos trescientos médicos cubanos vienen a Sudáfrica a trabajar en hospitales y clínicas situados 
en las áreas más remotas del país. La Facultad de Veterinaria de la University of Pretoria (UP) - la única en Sudáfrica- a menudo tiene estudiantes de posgrado procedentes de Hispanoamérica.

Las universidades sudafricanas cuentan con apoyo y ayudas de las misiones diplomáticas del mundo hispánico. La ayuda de la Embajada de España y del Ministerio de Asuntos Exteriores se traduce en conceder becas a estudiantes sudafricanos para estudiar en universidades españolas, en nombrar a lectores españoles para la difusión de la lengua, en proporcionar libros y material de audio y en otorgar subvenciones para congresos y otros eventos. La Embajadas de Perú y de México ofrecen becas de estudio y en el caso de México se incluye un curso de perfeccionamiento de la lengua. Todas las embajadas de Hispanoamérica - Argentina, Colombia, Cuba, Chile, México, Paraguay, Perú, Uruguay y Venezuela- ofrecen apoyo en forma de participación activa en eventos culturales en colaboración con las universidades sudafricanas.

Unisa posee el privilegio de disfrutar de un apoyo especial del sector privado en forma de becas ofrecidas por la empresa sudafricana-española Pescanova. A los mejores alumnos de cada curso se les otorga becas en efectivo y al graduado con nota de sobresaliente se le ofrece la oportunidad de asistir a un curso en España de un mes de duración.

\subsection{A nivel secundario}

El español, en contraste con el francés y el alemán, asignaturas tradicionales en el currículo secundario sudafricano, no se imparte oficialmente en ningún colegio o instituto de enseñanza media o superior. Sin embargo, sí forma parte de las lenguas extranjeras que el estudiante puede elegir para presentarse al examen de ingreso a la universidad. Las cifras arriba citadas demuestran que el número de candidatos de español va aumentando poco a poco. Este fenómeno es resultado del incremento en el número de familias diplomáticas hispanoparlantes asentadas en Sudáfrica desde las primeras elecciones democráticas en 1994, cuyos hijos continúan sus estudios en colegios sudafricanos. También están llegando a Sudáfrica más y más hombres y mujeres de negocios cuyos hijos han estudiado el español anteriormente en colegios en otras partes del mundo y desean mantenerlo. Ya que es obligatorio que cada candidato conozca por lo menos dos idiomas en su examen final, y recordemos que uno de ellos tiene que ser idioma oficial del país, la mayoría de estos candidatos optan por el inglés y el español. 
Durante el período 1973-1978, cuando el número de españoles residentes en Sudáfrica osciló en torno a 2.200, el Instituto Español de Emigración envió a profesores procedentes del Ministerio de Educación y Ciencia para impartir clases de español a los hijos de familias emigrantes españolas en las ciudades de Johanesburgo, Ciudad del Cabo, Port Elizabeth y Saldanha. La progresiva reducción del número de españoles residentes hizo que se suspendiera el programa y nunca ha sido reinstituido ${ }^{6}$.

\subsection{Institutos / Academias de lengua}

Las Universidades de Sudáfrica (Unisa), Witwatersrand (WITS) y Ciudad del Cabo (UCT) son las únicas que ofrecen cursos extracurriculares en español, destinados a principiantes y estudiantes de nivel intermedio. Los cursos forman parte de los programas de extensión universitaria o, como también se conoce, de educación para adultos. Suelen ser cursos de corta duración - de seis semanas a cuatro meses- que se imparten por la tarde, después de las horas de trabajo. Expiden certificados de competencia en la lengua, pero no son acreditativos.

En cuanto a las iniciativas privadas, existen escuelas de lengua en las que se imparte español en Johanesburgo, con cuatro escuelas, en Ciudad del Cabo que tiene siete escuelas y en Durban que cuenta con dos academias. Según el director de The Latin Connection, una escuela situada en Ciudad del Cabo y en Johanesburgo, dedicada exclusivamente a la enseñanza de español, la demanda a nivel particular de clases de español ha ascendido a un $70 \%$ durante los siete años de su existencia. Ninguna de estas escuelas expide diploma o titulación acreditiva.

\subsection{DELE en Sudáfrica}

Gracias a la iniciativa del Consulado General de España en Sudáfrica y los esfuerzos de la escuela The Latin Connection en Ciudad del Cabo, los estudiantes de español pueden acceder a los exámenes del Diploma en Español como Lengua Extranjera (DELE). Los diplomas son títulos oficiales otorgados por el Instituto Cervantes. En 1999, el primer año en que les fue ofrecida la oportunidad, unos veinte candidatos se examinaron del nivel inicial. Los exámenes se realizan en el Consulado General de España en Ciudad del Cabo y en el 2000 se espera que también se puedan facilitar en Pretoria en la Embajada de España. Por el momento sólo se ofrecen exámenes a nivel inicial y básico. 


\section{Quo Vadis: el español y el Hispanismo en Sudáfrica}

\section{Proyectos para el futuro}

\subsection{Indicadores para el futuro del español provenientes del sector privado}

Sudáfrica es una democracia reciente y por lo tanto sus relaciones comerciales, políticas y culturales con los países de habla hispana aún están en vías de desarrollo. Confiamos en que estos vínculos, según se vayan estrechando y fortaleciendo, resonarán favorablemente en el futuro del español y del hispanismo en este país. Veamos algunos de los indicadores que nos parecen los más prometedores:

Las relaciones comerciales entre Sudáfrica y España e Hispanoamérica han ido aumentando progresivamente desde 1994. En el año 1998, por ejemplo, España fue el undécimo mercado de exportación de productos sudafricanos. El comercio total entre ambos países (importaciones y exportaciones) durante 1998 aumentó en un 37\% con respecto a 1997, pasando de 111106 millones de pesetas en 1997 a 151986 millones de pesetas en 1998 (Sudáfrica Exporta, 1999: n 120). Las áreas de intercambio comercial entre Sudáfrica e Hispanoamérica por orden son: Argentina, México, Venezuela, Chile, Perú, Uruguay, Colombia y Paraguay. En estas relaciones se destaca la importancia de la minería. Entre las empresas sudafricanas con operaciones mineras en Latinoamérica figura la compañía de más prestigio del país -Anglo American-, la cual subvenciona el antes aludido puesto de investigador en asuntos de la América Latina en el Instituto de Relaciones Internacionales de la universidad WITS.

Estas estadísticas se traducen en una mayor visibilidad de productos y servicios españoles e hispanoamericanos entre la población sudafricana y en el consecuente aumento en el número de viajeros ejecutivos de y a Sudáfrica. El restablecimiento del servicio aéreo Johanesburgo-Madrid de Iberia -interrumpido entre los años 1986 y 1998 por motivos políticos y económicos - ha representado un incremento casi vertiginoso de turistas españoles a Sudáfrica y viceversa. Las aerolíneas sudafricanas (South African Airways) ofrecen servicios a Buenos Aires, mientras que Aero México y Aerolíneas Mexicanas y Aero Caribe comunican con toda la América Hispana. Estas compañías han registrado un incremento en el tráfico de turistas entre Sudáfrica y los países del Cono Sur y México, en particular.

Si a estos indicadores añadimos el aumento desde 1994 en el número de misiones diplomáticas hispánicas en Sudáfrica y de misiones sudafricanas en los países hispanoparlantes, prevemos que tendrán un impacto 
tarde o temprano en la demanda del aprendizaje de la lengua española. Una de las industrias más prometedoras para la economía sudafricana en el próximo futuro es el eco-turismo. La introducción de cursos de turismo y hostelería en las universidades e instituciones politécnicas proyecta la inclusión de cursos de español y de estudios sobre la cultura hispánica.

La frase «Roma no se construyó en un día» puede aplicarse al interés entre los sudafricanos por el mundo hispánico. No olvidemos que los años de aislamiento del mundo exterior todavía resuenan en Sudáfrica. Esto, unido a la tradición histórica de formar lazos con Gran Bretaña, sin hablar de la influencia de los Estados Unidos en cuanto a la cultura popular y los medios de comunicación masiva, no han dejado mucho lugar para mirar hacia los países hispanoparlantes hasta hace poco. Una de las señales de un cambio de enfoque se presenta en la prensa sudafricana en la que, con suma regularidad, aparecen artículos sobre uno u otro país hispánico. Los sudafricanos se están dando cuenta de que su proceso de transformación política, socio-económica y racial tiene paralelos con muchos países de habla hispana.

\subsection{Indicadores para el futuro del español provenientes del sector público y educativo}

La situación actual de la enseñanza de lenguas extranjeras no parece idónea en Sudáfrica y ya ha impactado negativamente en la del español, como señalamos en el apartado 4.1. Sin embargo, creemos que los cambios que se están introduciendo en el sistema educativo van a influir favorablemente en una creciente demanda del español y de los estudios hispánicos en el futuro. El gobierno del ANC se ha comprometido a transformar la educación en todos los niveles con el fin de resolver las injusticias y desigualdades del sistema de apartheid y de adiestrar a la población sudafricana de acuerdo con las necesidades del país. Esto no se limita a la educación de los jóvenes, sino que también afecta al $40 \%$ de los adultos sudafricanos que son o analfabetos o semianalfabetos. Por eso, con más frecuencia se enfoca a programas de estudio cuyos objetivos tienen una relación estrecha con una u otra vocación u orientación profesional. Y más allá del campo laboral, la intención educativa del gobierno es inculcar en sus ciudadanos el anhelo por aprender y fomentar la sensibilidad estética y cultural (Documento de SAQA: www.saqa.org.za/docs).

Con el fin de cumplir con estos objetivos y para promover el estudio del español y del hispanismo en Sudáfrica, los responsables del español 


\section{Quo Vadis: el español y el Hispanismo en Sudáfrica}

de los currículos a nivel terciario y secundario están promoviendo cambios importantes. De acuerdo con el nuevo plan de estudio, los alumnos de secundaria se prepararán para los exámenes preuniversitarios centrándose en el aspecto comunicativo del idioma, tanto en su forma hablada como escrita. Para crear una mayor concienciación por parte de los alumnos sudafricanos hacia el mundo hispánico y por parte de los hispanoparlantes hacia Sudáfrica, el plan les ofrecerá la oportunidad de hacer estudios comparativos entre un país hispánico y Sudáfrica en una diversidad de áreas como, por ejemplo, las costumbres, la cultura, el arte, la música, la historia, la economía y la política. Y con motivo de inculcar en los alumnos el amor por la literatura hispánica, se les proporcionará un libro de lecturas amenas y enriquecedoras con guías de lectura y sugerencias para otras lecturas. En un futuro no tan lejano, se proyecta que estos alumnos ubicados en diversas partes de Sudáfrica podrán acceder al conjunto de redes Internet para comunicarse con un profesor/tutor y para formar grupos de compañeros de curso con el fin de resolver problemas y realizar tareas en equipo.

La enseñanza electrónica promete ser un complemento imprescindible para la educación a distancia de la Universidad de Sudáfrica (Unisa) y su subdepartamento de español no se queda a la zaga en este área educativa. A partir de 1999 una amplia gama de material de instrucción en los cursos de español se dispone en la red de Unisa (www.unisa.ac.za/dept/). El próximo paso es impartir por vía electrónica la totalidad de cada uno de los nueve módulos semestrales de español a nivel pregrado y los ocho módulos semestrales de postgrado: el material de instrucción, la serie de tareas, la comunicación interactiva con el profesorado y los exámenes, tanto escritos como orales. La enseñanza a distancia tradicional -la palabra escrita enviada por correo- será un medio de instrucción estable en Unisa por muchos años ya que la mayoría de sus alumnos en Sudáfrica no dispone de los recursos informáticos para acceder a Internet. Sin embargo, la primera «cafetería» de ordenadores acaba de construirse cerca de Johanesburgo y se ha planificado la creación de muchas más a lo largo del país para ofrecer a todos los estudiantes la oportunidad de estudiar electrónicamente.

Junto con la aceleración en cuanto a los medios de enseñanza, el contenido de muchos programas de estudio también refleja las transformaciones en el sistema educativo sudafricano. Como materia de estudio independiente en Unisa, el español ofrece el programa de estudios de lengua, literatura y cultura para responder a los objetivos generales de la comunicación y el desarrollo intelectual y afectivo del individuo. Y dentro de éstos se encuentran los objetivos específicos de conocer la lengua para 
poder emplearla en una variedad de situaciones y circunstancias y de entender y evaluar la cultura y la literatura hispánicas para poder apreciar sus valores estéticos, intelectuales y afectivos. La oferta de la disciplina del español en módulos semestrales hace posible que esté incluida en muchos otros planes de estudios como, por ejemplo, el de turismo, de relaciones internacionales, de traducción, etc., que forman componentes no sólo del grado BA sino también de otros grados en Ciencias Económicas y Empresariales, Educación y Pedagogía, Teología y Derecho.

No se pueden negar las limitaciones presentadas por el hecho de que el español como disciplina universitaria aparece en una sola universidad en toda el África meridional. La falta de contacto con colegas de español y la consecuente ausencia de medidas de comparación producen el efecto de trabajar en un vacío. Gracias a Internet, al correo electrónico y, muy importante, a la reciente apertura de Sudáfrica al mundo académico internacional, poco a poco se están venciendo estos obstáculos. Otra avenida prometedora es el interés que algunas universidades y centros del mundo hispánico han empezado a mostrar por establecer convenios con Unisa y otras universidades sudafricanas para promover el intercambio de personal y estudiantes y para establecer una mayor cooperación en determinadas áreas de investigación. La Universidad de Alcalá, por ejemplo, está en proceso de firmar un convenio de cooperación e intercambio académico con Unisa para realizar proyectos de interés mutuo. En Argentina se ha fundado un centro de acceso para estudiantes de Unisa en ese país.

\section{Palabras de conclusión}

En la opinión de las autoras de este ensayo, el potencial del hispanismo en Sudáfrica es grande. Los indicadores señalan que el proceso de apertura de Sudáfrica al mundo más allá de sus fronteras y las transformaciones en el sistema educativo van a traducirse en un reconocimiento de la necesidad de las lenguas extranjeras en general y de la del español en particular. La cuestión ahora es cómo estimular este potencial y acelerar su ritmo. Tal trabajo sería algo más fácil si en Sudáfrica se contara con un Instituto Cervantes o con otra organización encargada de ayudar a difundir la cultura y la lengua hispánicas. Sin embargo, y dadas las limitaciones presupuestarias de los pocos individuos dedicados a la promoción del hispanismo en Sudáfrica, se pueden proyectar algunas iniciativas. Las palabras claves son la colaboración y la repartición de recursos. En el área educativa esto significa que un es- 


\section{Quo Vadis: el español y el Hispanismo en Sudáfrica}

tudiante matriculado en cualquier universidad o instituto terciario politécnico en Sudáfrica o en los países vecinos podrá estudiar módulos acreditativos de español en Unisa, sin perjudicar su titulación final. A nivel secundario y en los centros privados la preparación formal de profesores de español como lengua extranjera en Unisa ayudará a mejorar la calidad del profesorado en el país. La promoción de los exámenes oficiales del DELE en Sudáfrica es otro factor que añadir al estatus de la lengua y de sus aprendices. La formación de una asociación de hispanistas, por pequeña que sea al principio, se prevé como un apoyo no sólo a los profesores de español en todos los niveles, sino también como una oportunidad para intercambiar ideas que llevarán al mayor desarrollo del español y del hispanismo en Sudáfrica. Con la buena voluntad y la determinación de vencer obstáculos que caracterizan a la sociedad sudafricana, confiamos en que el futuro del hispanismo en Sudáfrica está asegurado.

\section{Notas}

1 El resumen está basado en una ponencia del profesor Andries Oliphant presentada en el congreso de AALRDISA (The All African Languages Re-development Instititute for Southern Africa) el 30 de julio de 1999, en el informe de Victor Webb 1995 Language in South Africa y en Towards a National Language Plan for South Africa, 1996.

2 Ésta y las demás estadísticas son aproximadas. Nuestras fuentes han sido las embajadas de procedencia, asociaciones representativas de las mencionadas comunidades y el libro International Trade Statistics Yearbook.

$3 \mathrm{El}$ árabe moderno que se usa en el Medio Oriente, a lo largo del mundo musulmán y en el Occidente en los medios de comunicación masiva (Mohammed, 1997:1).

4 Las siglas de las universidades sudafricanas mencionadas: PUCHE (Potchefstroom University for Christian Higher Education), RAU (Rand Afrikaans University, Johanesburgo), RU ( Rhodes University, Grahamstown), UCT (University of Cape Town), UDW (University of Durban-Westville), UN (University of the North, Sovenga), UND (University of Natal, Durban), UNP (University of Natal, Piertersmaritzberg), Unisa (University of South Africa, Pretoria), UNITRA (University of the Transkei, Umtata), UOFS (University of the Orange Free State, Bloemfontein), UP (University of Pretoria), UPE (University of Port Elizabeth), US (University of Stellenbosch),UWC (University of the Western Cape, Belleville), UZ (University of Zululand, Empangeni),WITS (University of the Witwatersrand, Johanesburgo). Las estadísticas y otros datos nos han sido proporcionados por el personal académico y administrativo de estas universidades a quienes quedamos agradecidas.

5 Agradecemos al Dr F Calitz del South African Certification Council, al Dr C Lotter del South African Universities Vice Chancellors' Association y al Sr W Venter de Department of Education el acceso a estas estadísticas.

6 Agradecemos al Sr Nabor García, Cónsul General de España en Sudáfrica, por su gentileza en proporcionarnos estos datos. 


\section{Bibliografía}

Government Gazette, 9 de mayo de 1997. Publication of language in education documents with the the intention to invite comments from the public. Volume 383. Number 17997

International Trade Statistics Yearbook (Volume I) 1997 New York: United Nations

KADT, E. DE 1999 Learning and teaching foreign languages in tertiary institution in South Africa. Part 1: Towards a profile of language learners. Artículo que se publicará en Journal for Language Teaching.

LAPUerta Amigo, P. 1997 La enseñanza del español en la nueva Sudáfrica: Problemas, soluciones y futuro. Actas de la Asociación para la Enseñanza del Español como Lengua Extranjera: $487-495$

MEYER, D. 1998 What teachers say they want, what they actually do and its implications for language in education policy. Journal for Language Teaching 32(1): 1-17.

Mohammed, Y. 1997 The Teaching of Arabic in South Africa. Belleville: University of the Western Cape

SAQA (South Africa Qualifications Authority) Documento AThe Seven Critical Outcomes and The Five Citizenship Oucomes@ (http://www.saqa.org.za/docs/)

Sudáfrica Exporta 1999 No 120 (Septiembre). Madrid: Sección Económica, Embajada de Sudáfrica (http://www.sudafrica.com/sudafricaexporta/noticia1.htm)

Towards a National Language Plan for South Africa. 1996 The final report of the Language Plan Task Group (LANTAG) 1996. Pretoria: Department of Arts, Culture, Science and Technology.

WebB, V. 1995 Language in South Africa. An input into language planning for a postapartheid South Africa. Pretoria: University of Pretoria (The Licca Research and Development Programme). ${ }^{\circ}$ 